Trauma Berufskrankh 2008 10

[Suppl 2]:259-262

DOI 10.1007/s10039-008-1366-0

Online publiziert: 19. April 2008

(c) Springer Medizin Verlag 2008

\author{
H. Krimmer \\ Zentrum für Handchirurgie, Elisabethenkrankenhaus, Ravensburg
}

\title{
Neues und Zukünftiges in der Versorgung von Radiusfrakturen
}

\section{Präoperative Vorbereitung}

Die Behandlung der distalen Radiusfraktur hat in den letzten Jahren eine massive Trendwende weg von der konservativen hin zur operativen Behandlung erfahren. Die sekundäre Dislokation nach initial gutem Repositionsergebnis und die unzureichende Wiederherstellung der Gelenkfläche mit geschlossener Reposition bei intraartikulären Frakturen führten häufig zu unbefriedigenden klinischen Ergebnissen. Die Anwendung der KirschnerDraht-Fixierung und des Fixateurs externe konnten den Anforderung der Reposition und dauerhaften Retention nur unzureichend gerecht werden. Die Plattenosteosynthese ging insbesondere bei dorsaler Trümmerzone und intraartikulären Frakturen mit der Schwierigkeit einher, dass Standardschrauben in der distalen Trümmerzone keinen Halt finden und meist zusätzlich eine Spongiosaplastik notwendig wird. Hinzu kam die Problematik, dass das Plattendesign den Anforderungen der atraumatischen Reposition und Retention nicht gerecht werden konnte und dass Narbenprobleme und Ausheilung in Fehlstellungen häufige Probleme darstellten.

Mit der Einführung der winkelstabilen Platten konnten viele dieser Schwierigkeiten gelöst werden. Die palmare winkelstabile Plattenosteosynthese stellt mittlerweile das bevorzugte Osteosyntheseverfahren dar und ermöglicht insbesondere bei intraartikulärer Beteiligung eine anatomische dauerhafte Retention der Gelenkfläche. Allerdings müssen einige grundlegende Aspekte beachtet werden, um mit dieser Technik zum Erfolg zu kommen und ernsthafte Komplikationen zu vermeiden.
Nach Diagnosestellung durch konventionelle Röntgenaufnahmen stellt die ergänzende CT-Untersuchung mit $3 \mathrm{D}$-Rekonstruktion bei intraartikulärer Beteiligung eine wertvolle Hilfe zur Planung des operativen Vorgehens dar (- Abb. 2c). Bei der so genannten Die-Punch-Fraktur mit zentraler Impression der Gelenkfläche sollte sie zwingend vorliegen, da hier mit konventionellen Aufnahmen die Beurteilung der Fraktursituation nicht möglich ist (- Abb. 1a).

\section{Operation}

\section{Zugang}

Der palmare Zugang stellt die bevorzugte Methode dar, da er eine exakte Reposition und Kontrolle ermöglicht und die Platte mit guter Weichteildeckung platziert werden kann. Um Komplikationen mit lang anhaltenden, u. U. dauerhaften Problemen zu vermeiden, sollte unbedingt radial des N. medianus zwischen M. flexor carpi radialis und A. radialis der $\mathrm{M}$. pronator quadratus dargestellt und abgelöst werden (Henry-Zugang). Eine Schnittführung ulnar des N. medianus, die in der Regel direkt über diesem zu liegen kommt, ist in der Regel durch schmerzhafte Narbenprobleme um den Nerv gekennzeichnet. Hinzu kommt, dass der Nerv durch die eingesetzten Homann-Haken nach radial gedrückt wird, mit der Folge von Druckschäden und nachfolgenden Dys- und Hyperästhesien, die das klinische Ergebnis nachhaltig trüben können.
Muss die Inzision bei ausgeprägter Trümmerzone nach distal verlängert werden, sollte dies bevorzugt gewinkelt nach radial erfolgen. Eine gerade Verlängerung über die Handgelenkbeugefalte geht in der Regel ebenfalls mit einer erheblichen Narbenproblematik einher. Eine grundsätzliche Notwendigkeit der Karpaldachspaltung sehen wir nicht, allerdings sollte präoperativ nach Sensibilitätsstörungen gefragt werden. Werden diese bejaht, sollte der Karpalkanal durch gesonderte klassische Schnittführung in der Interthenarfurche eröffnet werden.

Nach Ablösen des M. pronator quadratus empfiehlt es sich gerade bei intraartikulären Frakturen, die häufig auch den Processus styloideus radii einbeziehen, das 1. Strecksehnenfach zu eröffnen und den Ansatz des M. brachioradialis abzulösen, da hieraus häufig ein Repositionshindernis resultiert. Gelingt die Reposition von palmar nicht vollständig, kann ergänzend ein limitierter dorsaler Zugang benutzt werden. Zentrale Impressionsfrakturen müssen in der Regel durch einen solchen versorgt werden mit Hochstößeln der Gelenkfläche und ggf. Abstützung durch Spongiosaplastik (- Abb. 1b).

\section{Osteosynthesematerial}

Grundsätzlich sollten winkelstabile Implantate verwendet werden, da bei intraartikulären Frakturen in der distalen Trümmerzone keine stabile bikortikale Schraubenverankerung möglich ist. Bevorzugt kommen Low-Profile-Platten mit 2 distalen Reihen und mit Schraubendurchmessern von 2,4-2,7 mm zur Anwendung, um 


\section{Radiusfraktur}

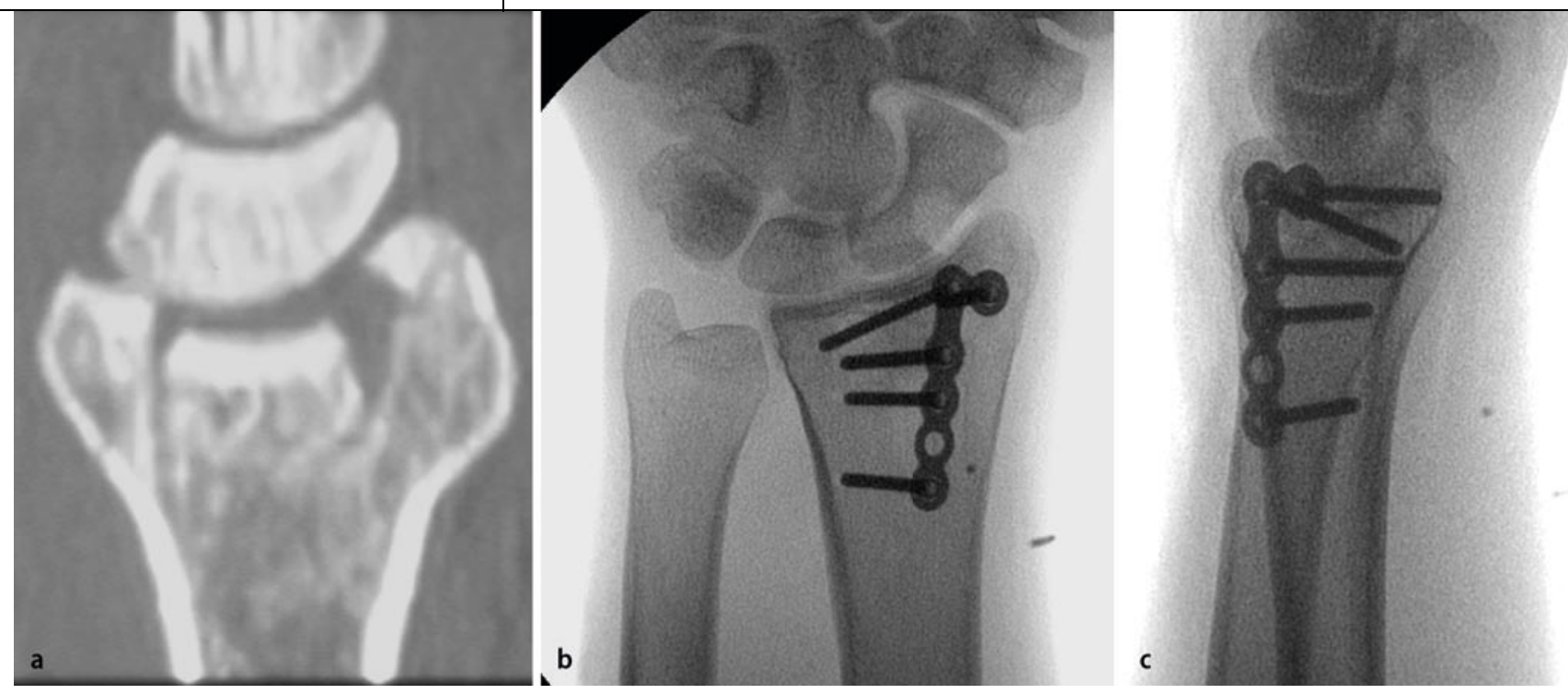

Abb. $1 \Delta$ a Zentrale Impressionsfraktur (Die-Punch-Fraktur), b Stabilisierung von dorsal mit winkelstabilem 2,5-mm-Miniimplantat (Aptus ${ }^{\circledast}$ von Medartis)
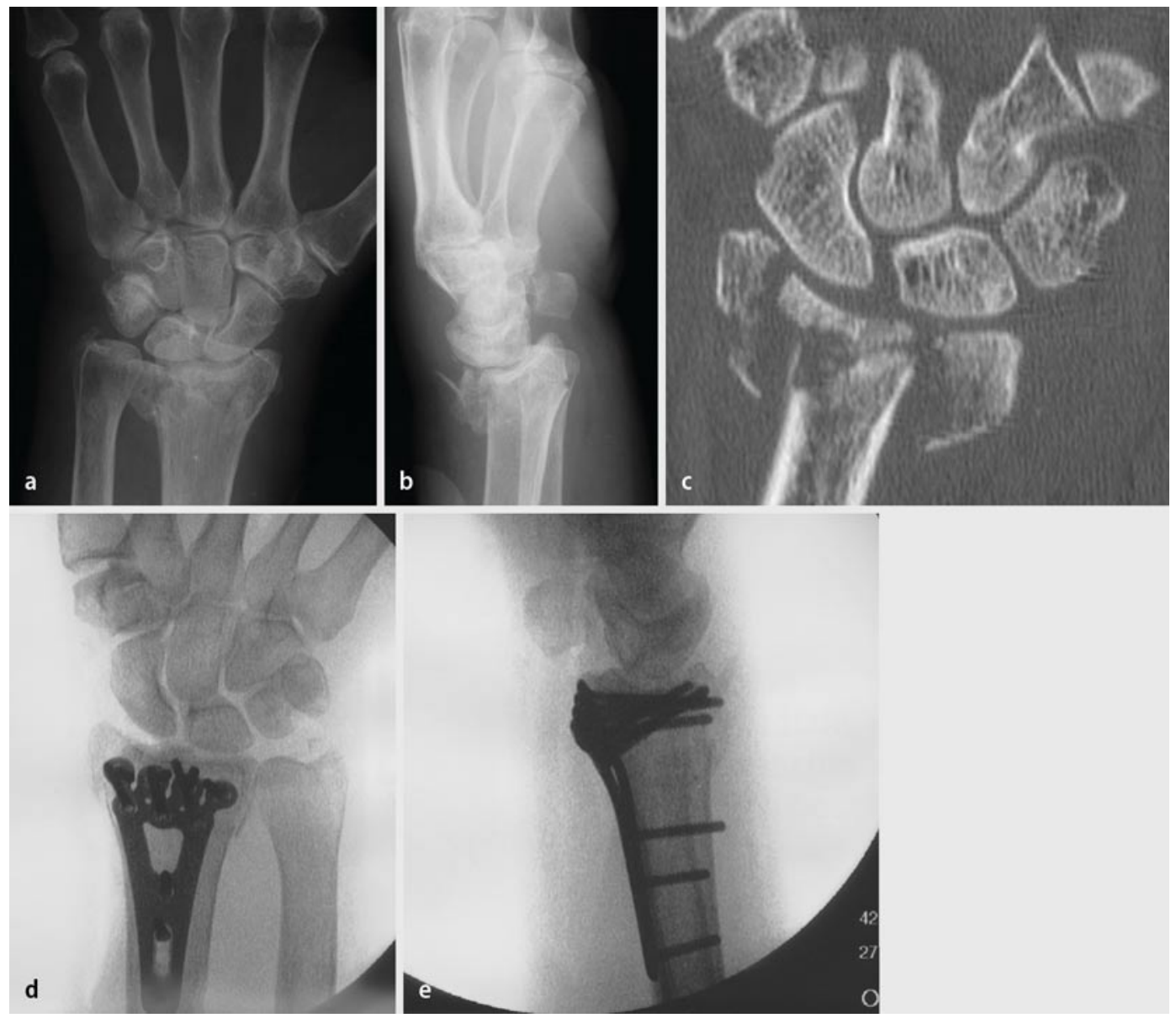

Abb. 2 < 75-jähriger Patient mit Radiustrümmerfraktur, a,b 1 Woche nach konservativer Behandlung, c Analyse im CT mit radialer und ulnarer Gelenkflächenbeteiligung, $\mathbf{d}, \mathbf{e}$ Stabilisierung durch polyaxiale winkelstabile 2,5-mm-Platte (Aptus ${ }^{\circledast}$ von Medartis) mit zentraler und dorsaler $\mathrm{Ab}$ stützung der Gelenkfläche

eine feine Abstützung der einzelnen Gelenkflächenanteile zu gewährleisten. Die multidirektionale Schraubenplatzierung erlaubt in Verbindung mit den 2 distalen Reihen eine stabile zentrale und dorsale Unterstützung der Gelenkfläche. Darüber hinaus kann die Schraubenplatzie- rung der Fraktur angepasst werden - mit besonderer Berücksichtigung der Problematik des ulnaren Kantenfragments und Frakturen des Processus styloideus radii. Dies ist bei unidirektionalen Systemen nur bedingt möglich.

\section{Reposition und Fixierung}

Nach Darstellung der Fraktur über den palmaren Zugang erfolgt durch Zug und Flexion mit Druck von dorsal die Reposition. So bald die Gelenkfläche kongruent steht und die Radiuslänge hergestellt 
ist, wird die Platte in einem proximalen Gleitloch fixiert. Unter dem Bildwandler wird die Lage kontrolliert, mit besondere Beachtung der zentralen achsengerechten Lokalisation und des distalen Endes. Der distalen Umschlagslinie am Radius, der so genannten „water-shed line“, kommt dabei eine besondere Bedeutung zu, da die Platte nicht weiter distal liegen sollte. Bei Nichtbeachtung besteht die Gefahr, dass an dem dann frei liegenden Plattenrand Beugesehnenrupturen entstehen können. Im Gleitloch kann die Platte nach distal oder proximal variiert werden. Danach wird sie proximal mit einer 2. Schraube fixiert und dient jetzt als Abstützung für die definitive Reposition und Fixierung. Unter Flexion und Zug werden jeweils eine Schraube aus der 1. und 2. Reihe eingebracht. Nach erneuter Röntgenkontrolle werden die restlichen Schrauben platziert. Zum sicheren Ausschluss intraartikulärer Schraubenlagen sollte in der p.-a. Projektion leicht schräg entsprechend der palmaren Inklination durchleuchtet werden. Auch hier ist bei Nachweis einer intraartikulären Position ein multidirektionales System von Vorteil, da die Schraubenrichtung unter Belassen der Platte problemlos geändert werden kann ( $\bullet$ Abb. 2).

Gelingt die Reposition initial mit dieser Technik nicht, kann der Zugang gewinkelt nach radial erweitert werden. Wenn das 1. Strecksehnenfach eröffnet und der Ansatz des M. brachioradialis abgelöst sind, können der Radiusschaft herausgedreht und die Radiusgelenkfläche unter Sicht von proximal reponiert werden. Ergänzend kann zur Kontrolle das Radiokarpalgelenk durch Inzision der radiokarpalen Bänder im Faserverlauf eröffnet werden. Ist das Repositionsergebnis instabil, empfiehlt sich eine passagere Kirschner-Draht-Fixierung von radial oder ulnar, alternativ auch durch spezielle Löcher in der Platte.

Stehen die radiale oder ulnare Seite im Vordergrund, sollte die Platte in diesem Bereich mit mindesten 3 Schrauben fixiert werden. Quere gesonderte Zugschrauben von radial Richtung Ulnakopf sind problematisch, da sie ein hohes Gefahrpotenzial für Knorpelschäden im distalen Radioulnargelenk darstellen. Durch gezielte Schraubenplatzierung von palmar kann der Processus styloideus radii nach passagerer Kirschner-Draht-Fixierung stabilisiert werden ( $\bullet$ Abb. 2).

Gelingt die Reposition allein von palmar nicht, muss zusätzlich ein dorsaler Zugang zur Reposition benutzt werden, u. U. mit der Technik der Doppelplattenosteosynthese. Bei Zertrümmerung der Gelenkfläche sehr weit distal ist die Retention mit Platten häufig nicht ausreichend möglich, hier kann von der Verwendung einzelner Minifragmentschrauben, die meist einen erstaunlich guten Halt finden, Gebrauch gemacht werden. Zentrale Impressionsfrakturen werden nach Reposition über einen dorsalen $\mathrm{Zu}$ gang durch winkelstabile Miniimplantate fixiert.

Bei ausgeprägter Weichteilschädigung empfiehlt sich der Einsatz eines Fixateur externe mit später additiver offener Reposition und Plattenosteosynthese. Die Technik der Kirschner-Draht-Osteosynthese bei intraartikulären Frakturen führt meist zu unbefriedigenden Ergebnissen.

\section{Begleitverletzungen}

Intraartikuläre Frakturen haben eine erhöhte Inzidenz für behandlungsbedürftige Begleitverletzungen. Nach Stabilisierung der Radiusfraktur sollte man daher grundsätzlich auf eine skapholunäre Bandruptur (SL-Dissoziation) und eine Instabilität im distalen Radioulnargelenk überprüfen. Unter Bildwandlerkontrolle werden in p.-a. Projektion dynamisch der skapholunäre Spalt und die Kinematik des Skaphoids geprüft. Bei Aufweiten des Spalts über $3 \mathrm{~mm}$ und ggf. erweitertem SL-Winkel in der seitlichen Projektion von mehr als $70^{\circ}$ besteht der dringende Verdacht auf eine SL-Bandläsion, die eine Reposition und passagere KirschnerDraht-Fixierung erfordert. Die Stabilität im distalen Radioulnargelenk wird manuell geprüft, bei eindeutiger Luxationstendenz sind eine Refixierung des Processus styloideus ulnae oder bei fehlender Fraktur eine transossäre Refixierung des ulnokarpalen Bandapparats erforderlich.

\section{Postoperatives Regime}

Das Handgelenk sollte nach der Operation durch eine palmar angelegte Schiene stabilisiert werden. Dies ist v. a. wegen
Trauma Berufskrankh 2008 · 10

[Suppl 2]:259-262

DOI 10.1007/s10039-008-1366-0

(C) Springer Medizin Verlag 2008

\section{H. Krimmer}

\section{Neues und Zukünftiges in der Versorgung von Radiusfrakturen}

\section{Zusammenfassung}

Die operative Behandlung der distalen Radiusfraktur hat mit Einführung der winkelstabilen Implantate einen enormen Auftrieb erhalten. Die perioperative Morbidität ist durch die stabile Fixierung und kürzere Ruhigstellung mit Frühmobilisierung reduziert. Aus Sicht des Chirurgen ist die operative Versorgung auch bei schwierigen intraartikulären Frakturen mit dieser Technik erleichtert. Allerdings stellt diese Methode keine Erfolgsgarantie dar und bedarf daher der exakten Planung, um bisher kaum bekannte Komplikationen wie Beugesehnenrupturen oder intraartikuläre Schraubenlagen zu vermeiden. Polyaxiale Low-Profile-Implantate, die der jeweiligen Fraktursituation angepasst werden können, stellen die neueste Generation der verwendeten Platten dar. Osteointegrative Implantate werden die Zukunft bestimmen.

\section{Schlüsselwörter}

Radiusfraktur · Winkelstabiles Implantat · Polyaxiale Schrauben - Low-Profile-Implantat . Osteointegratives Implantat

\section{Innovations and future aspects in the treatment of distal radius fracture}

\section{Abstract}

The treatment strategy of distal radius fractures has significantly changed with the use of fixed angle devices. From the patient's point of view, perioperative morbidity has decreased; from the surgeon's point of view, the palmar approach facilitates reduction and fixation even in severe comminuted fractures. However, this method needs precise practice to avoid complications rarely seen in the past, such as flexor tendon ruptures and intraarticular screw concerns. Low-profile polyaxial plates that are adjustable according to fracture type represent the new generation. Osteointegrative materials are the goal for the future.

\section{Keywords}

Distal radius fracture - Fixed angle device $\cdot$ Polyaxial screws - Low-profile plate · Osteointegrative material 
der Beübung der Handfunktion wichtig, da ein kompletter Faustschluss und die komplette Fingerstreckung rasch möglich sein müssen, um lang währenden Störungen vorzubeugen. Hinzu kommt, dass Teilrupturen des ulnokarpalen Komplexes und karpalen Bandapparats eine Mindestruhigstellungszeit zur Ausheilung benötigen, um nicht später längerfristig Probleme zu bereiten. Für intraartikuläre Frakturen wählen wir daher eine Ruhigstellung von 4 Wochen, wobei das Handgelenk nach 2 Wochen aus der Schiene heraus mobilisiert wird. Extraartikuläre Frakturen werden 2 Wochen ruhig gestellt.

Störungen der Handfunktion werden nicht selten vernachlässigt und erfordern den frühzeitigen handtherapeutischen Einsatz. Durch diese Maßnahmen und eine adäquate postoperative Analgesie und Schwellungsprophylaxe durch Hochlagern und Eisapplikation kann die Inzidenz einer Heilentgleisung im Sinne eines CRPS (komplexes regionales Schmerzsyndrom) auf ein Minimum reduziert werden.

\section{Fazit}

Die winkelstabile Plattenosteosynthese ermöglicht gerade bei intraartikulären Radiusfrakturen eine stabile dauerhafte Fixierung mit anatomiegerechter Ausheilung und reduzierter Morbidität aus Patientensicht. Aus Sicht des Chirurgen wird der Umgang mit diesen schwierigen Verletzungen erleichtert. Allerdings müssen Grundprinzipien beachtet werden, um mit dieser Methode zum Erfolg zu kommen. Der Einsatz osteointegrativer resorbierbarer Osteosynthesematerialien, die die Stabilitätskriterien erfüllen und überschießende Narbenreaktionen vermeiden, ist ein Ansporn für weitere Forschungsvorhaben.

\section{Korrespondenzadresse}

\section{Prof. Dr. H. Krimmer}

Zentrum für Handchirurgie,

Elisabethenkrankenhaus Ravensburg,

Elisabethenstraße 17, 88212 Ravensburg

info@handchirurgie-ravensburg.de

Interessenkonflikt. Der korrespondierende Autor gibt an, dass kein Interessenkonflikt besteht.

\section{Literatur}

1. Krimmer $\mathrm{H}$, Pessenlehner $\mathrm{C}$, Hasselbacher $\mathrm{K}$ et al. (2004) Palmare winkelstabile Plattenosteosynthese der instabilen distalen Radiusfraktur. Unfallchirurg 107: 460-467

2. Orbay JL, Fernandez DL (2004) Volar fixed-angle plate fixation for unstable distal radius fractures in the elderly patient. J Hand Surg [Am] 29: 96-102 